\title{
Motion Control of a Three-Dimensional Eel-like Robot Without Pectoral Fins *
}

\author{
M. El Rafei, M. Alamir, N. Marchand* \\ M. Porez and F. Boyer ${ }^{* *}$ \\ * University of Grenoble \\ Control system department of GIPSA-Lab, Grenoble, France \\ (maher.el-rafei, mazen.alamir, nicolas.marchand@lag.ensieg.inpg.fr) \\ ** Institut de Recherche en Cybernétique de Nantes, France \\ (mathieu.porez@irccyn.ec-nantes.fr, frederic.boyer@emn.fr)
}

\begin{abstract}
In this paper, recent advances in the design of feedback laws for the 3D movement of an Eel-like robot are presented. Such a robot is under construction in the context of a national French robotic project. The proposed feedback enables the tracking of a desired 3D position of the Eel head as well as the stabilization of the rolling angle without using pectoral fins. We build on a previous work in which we proposed a complete control scheme for robot's 3D movement using its pectoral fins. The controller is tested on a recently developed complete 3D model in order to assess its efficiency in tackling 3D manoeuvres.
\end{abstract}

\section{INTRODUCTION}

In this paper, current researches on the control of an eel-like robot are presented. This is done in the context of a multidisciplinary French national research project called RoBeA-Anguille ${ }^{1}$. The aim of this project is to design, construct and control the 3D motion of an eel-like robot. The prototype under construction is obtained by connecting many parallel platforms (see figure 1). The eel's body will then be covered by a deformable "skin" in order to achieve high performance swimming. As it has been underlined by many researchers in the robotic biomimetic field, understanding the dynamics of such robots may be of a great interest in improving the manoeuvrability of under-water vehicles (see Triantafyllou et al. [1993], Mason and Burdick [2000], Morgansen et al. [2002], McIsaac and Ostrowski [1999], Colgate and Lynch [2004], Sfakiotakis et al. [1999], Triantafyllou and Triantafyllou [1995], Bandyopadhyay [2005]).

A 3D continuous model of the target prototype has been proposed by (Boyer et al. [2006]) using the geometrically exact theory of beams under finite deformations (Simo [1989]). This model is used here to validate the proposed $3 D$ control feedback.

There exist many works in the literature that studied the eel-like robots movements. In particular, (McIsaac and Ostrowski [1999] and the related works) have studied the 2D movement of an eel-like robot. The rolling cart analogy is used in order to derive state feedback that track some reference trajectories. Another interesting approach was proposed by Morgansen et al. [2001, 2002], Vela et al. [2002] where averaging formulas have been derived to describe the mean behavior over a cycle.

\footnotetext{
* This work was supported by the French National Center for Scientific Research (CNRS) in the context of the ROBEA-project and the French National research agency project (ANR-RAAMO).

1 http://www.irccyn.ec-nantes.fr/hebergement/ROBEA/
}

A design procedure for a biomimetic robot-fish based on improved kinematic propulsive model has been described by $\mathrm{Yu}$ et al. [2005] where the basic motion control laws were presented. For a detailed review of existing works on control of swimming, the reader can refer to Colgate and Lynch [2004].

However, few researches have studied the control methods for 3D motion of eel-like robots. Preliminary results on the 3D control of an eel-like robot are presented by Alamir et al. [2007] where a complete control scheme for 3D movement of the continuous model (Boyer et al. [2006]) was proposed. The motion and the velocity in the transverse plane are controlled by monitoring the oscillatory gait characteristics while the altitude changes and the rolling stabilization task are handled by means of two pectoral fins that are attached to the eel's head.

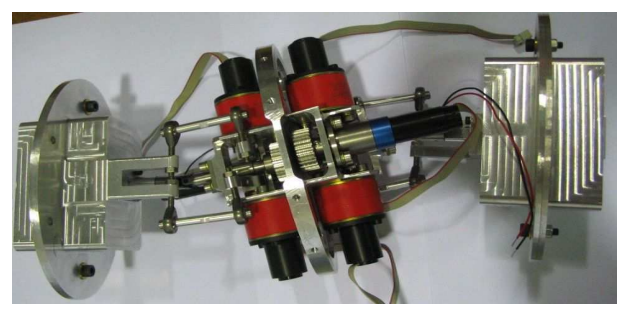

Fig. 1. An actuated link

The current work tackles another challenging direction; namely, the problem of 3D motion control without pectoral fins. In other words, the planar movement's strategy still the same while the altitude changes and the rolling stabilization task are handled only by 3D robot's body movements without using pectorals fins. Our basic concern while developing the control strategy was the simplicity and the robustness against modeling errors. To achieve this, very simple feedback laws have been derived that are quite independent of the structure of the simulator 
equations. The latter is only used to assess the performance of the proposed feedback laws. In other words, since the controller uses too few information about the simulation model, it is likely to work on the real system (probably after some tuning phase) even if it differs from the simulation model used here to assess its performance.

This paper is organized as follows : First, the mathematical model used in the simulator is briefly described in section 2. Since the latter is quite complex, only the related guidelines are briefly mentioned. Feasibility analysis of our control principle is briefly shown in section 3. Section 4 clearly states the $3 D$ control problem. The different "components" of the feedback law are then presented in section 5, namely, the control of the head position in the transverse plane, the control of the robot altitude as well as the rolling angle stabilization. Some $3 D$ scenarios are proposed in section 6 . The paper ends by some concluding remarks together with the road map for future works.

\section{THE MATHEMATICAL MODEL OF THE CONTINUOUS EEL-LIKE ROBOT}

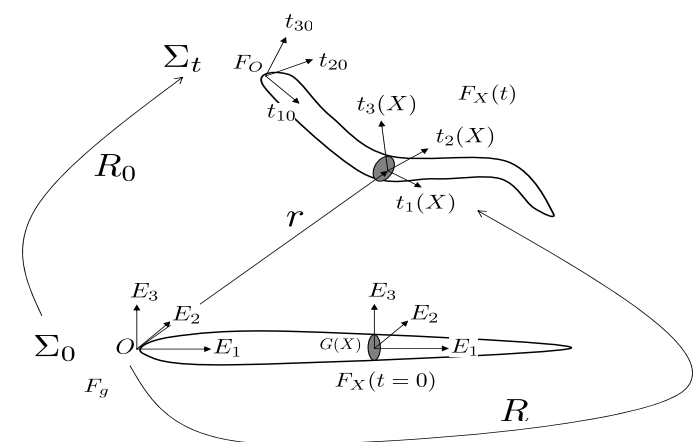

Fig. 2. Frames and parametrization of the continuous eel robot model

For a complete description of the underlying mathematical model, the reader is referred to the basic paper (Boyer et al. [2006]). Only the main features of the model in the non stretchable case are mentioned here in order to give an idea about the model complexity and how the $3 D$ features are handled.

Figure 2 illustrates the basic notations used in the description of the mathematical model. Let $X$ designates the material abscissa along the eel's mean line and $G(X)$ the center of mass of the $X$ section. Under the assumption of non-stretchable body, the configuration of the robot at instant $t$ (after deformation) is completely defined by the value at each $X \in[0, L]$ ( $L$ is the robot's length) of the rotation matrix $R(t, X)$ mapping the head basis before deformation to the basis at instant $t$ of the section situated at the abscissa $X$ (see figure 2).

Once given the rotation matrix and the position of the head , namely $R_{0}(t)=R(t, 0)$ and $r_{0}(t)=r(t, 0)$, the deformation of the body is completely defined by $\frac{\partial R}{\partial X}$ that can be written as follows :

$$
\frac{\partial R}{\partial X}=R \hat{K} \quad ; \quad R(t, 0)=R_{0}
$$

where $\hat{K}(t, X)$ is a skew symmetric tensor associated to an axial vector $K(t, X)$. Note that (1) is nothing but a change in the description variables since $\hat{K}=R^{T} \frac{\partial R}{\partial X}$ becomes the new d.o.f that defines the deformation of the eel's body. Note that the last two components of $K$, namely $K_{2}$ and $K_{3}$ stand for the curvatures of the beam in the two planes $\left(G, t_{1}, t_{3}\right)(t, X)$ and $\left(G, t_{1}, t_{2}\right)(t, X)$ while the first component $K_{1}$ stands for the torsion strain field.

It is important to note immediately that in the present paper, the vector field $K(\cdot, \cdot)$ is the control input. This assumes that the distributed actuators are conveniently used to produce the corresponding body deformation in within the allowable powers and excursions.

Using the above notations, the non stretching assumption can be expressed as follows :

$$
r^{\prime}=\frac{\partial r}{\partial X}=t_{1}(t, X) \quad ; \quad r(t, 0)=r_{0}(t) .
$$

Let us now introduce the field of angular velocities $\hat{\omega}(t, X)$ :

$$
\hat{\omega}=\dot{R} R^{T} \quad(\text { that is } \dot{R}=\hat{\omega} R) .
$$

The field $\hat{\omega}$ can also be represented by its axial vector $\omega$. It can be proved (see Cardona and Géradine [1988]) that :

$$
\frac{\partial \omega}{\partial X}=R \dot{K} \quad ; \quad \omega(t, 0)=\omega_{0}(t) .
$$

This means that, given the control $K(\cdot, \cdot)$ and the configuration $R(\cdot, \cdot)$, the integration of (4) in space enables the computation of $\omega$, hence $\hat{\omega}$ and therefore $\dot{R}$ thanks to (3).

On the other hand, by differentiating (2) in time, it comes that :

$$
\frac{\partial \dot{r}}{\partial X}=\omega \times t_{1} \quad ; \quad \dot{r}(t, 0)=\dot{r}_{0}(t),
$$

that can be integrated in order to reconstruct $\dot{r}(t, X)$ for all $X \in[0,1]$. Similarly, further derivations enables to write the second derivatives $\ddot{r}$ and $\dot{\omega}$ as functions of the head accelerations $\ddot{r}_{0}$ and $\dot{\omega}_{0}$, the velocities $\dot{r}_{0}, \omega_{0}$ and the time derivatives of the strains field $K$. This can be shortly written as follows :

$$
\left(\begin{array}{c}
\ddot{r} \\
\dot{\omega}
\end{array}\right)(t, X)=\Gamma\left(t, X, K(\cdot, \cdot), \dot{r}_{0}, \omega_{0}, \ddot{r}_{0}, \dot{\omega}_{0}\right) .
$$

Note that the map $\Gamma$ uses the control profile $K(\cdot, \cdot)$ in time and space through time derivations and integration over space as it has been done above for the computation of the velocities $\dot{r}$ and $\omega$. Note that (5) expresses only kinematic constraints. In order to built the dynamic model, the external forces due to the contact with the fluid have to be computed. Assuming that the gravity forces are compensated by internal "air tanks", the only external forces are those due to the interaction of the body with the fluid. To express these forces and torques, the contact model of Morison [1950], Burgess [1993] is used. This amounts to integrate the following quantities along the eel's body :

$$
\begin{aligned}
& \frac{d f_{\text {ext }}}{d X}=-\sum_{i=1}^{3} C_{1 i}\left[\left|V_{i}\right| V_{i}\right] t_{i}-\sum_{i=1}^{3}\left[C_{2 i} \gamma_{i}\right] t_{i}, \\
& \frac{d c_{e x t}}{d X}=-\sum_{i=1}^{3} C_{3 i}\left[\left|\Omega_{i}\right| \Omega_{i}\right] t_{i}-\sum_{i=1}^{3}\left[C_{4 i} \Xi_{i}\right] t_{i},
\end{aligned}
$$


where $V_{i}, \gamma_{i}, \Omega_{i}$ and $\Xi_{i}$ are the components on $t_{i}$ of $\dot{r}, \ddot{r}$, $\omega_{i}$ and $\dot{\omega}_{i}$ respectively, namely :

$\dot{r}=: \sum_{i=1}^{3} V_{i} t_{i} ; \ddot{r}=: \sum_{i=1}^{3} \gamma_{i} t_{i} ; \omega=: \sum_{i=1}^{3} \Omega_{i} t_{i} ; \dot{\omega}=: \sum_{i=1}^{3} \Xi_{i} t_{i}$ while $\left\{C_{j i}\right\}$ are coefficients depending on the mass per unit volume of the fluid, the shape and the size of the section (elliptic in our case) and the Reynolds number of the moving section in the fluid. Note that the first term of (6) accounts for the drag/lift forces applied on the section while the second term accounts for the added mass forces as given by Techet and Triantafyllou [2004]. The same angular related significations hold for the terms in (7).

\section{PROBLEM FEASIBILITY}

First of all, in accordance with the true eels behavior, only the component $K_{3}$ of the deformation field was used in (Alamir et al. [2007]) $\left(K_{1} \equiv K_{2} \equiv 0\right)$. Namely, at any time $t$, the body mean line entirely lies in the plane $\left(0, t_{10}(t), t_{20}(t)\right)$ (see figure 5$)$. The direction of this plane in space was controlled by robot's pectoral fins.

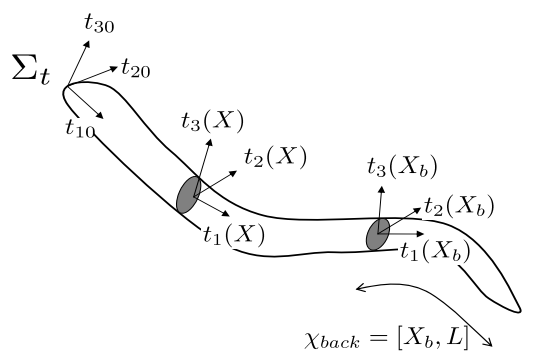

Fig. 3. Frames and parametrization.

The idea in the present work is to realize the altitude control without using the pectoral fins, by combining torsion (around $\left.t_{1}(X)\right)$ and pitch (around $\left.t_{2}(X)\right)$ movements with robot's undulatory movement (around $t_{3}(X)$ ). The principle consist of applying torsion and pitch movements to every back part section of the body in phase with its undulatory movement. This is motivated by the fact that the prototype under construction allows torsion, pitch and yaw movements for robot's vertebra. The robot's back part can be defined by :(See figure 3)

$$
\forall X \in \chi_{\text {back }}=\left[X_{b}, L\right]
$$

where $X_{b}$ is a given material abscissa and $L$ is the robot's length.

By doing so, the robot is divided into two parts. The front part's mean line entirely lies in the plane $\left(0, t_{10}(t), t_{20}(t)\right)$, while the mean line of the back part is used for control and can leave this plane $\left(0, t_{10}(t), t_{20}(t)\right)$.

Note that most fish swim forward by bending their bodies into a backward-moving propulsive wave that extends to its codal fin (tail). As the propulsive wave passes backward along the fish, each small body segment (called propulsion element) generate a force that increases the momentum of water passing backward (Webb [1984]). An equal opposing force $F$ (see figure 4 ) is subsequently exerted by the water on the propulsive element. $F$ is normal to this later and is analyzed into a lateral $F_{L}$ and thrust $F_{T}$ component.
In anguilliform swimming, since at least one complete wavelength of the propulsive wave is present along the body, lateral forces are adequately cancelled out, minimizing any tendencies for the body to recoil (Sfakiotakis et al. [1999]).

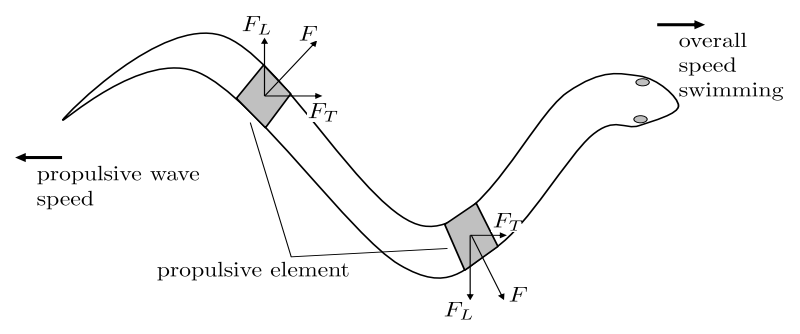

Fig. 4. Propulsive element force generation

Since at least one complete wavelength of the propulsive wave is present along the body, the analysis of different forces exerted on the robot's two parts permit to see the following effects :

- The application of a sine twist movement to the robot's back part :

$$
\forall X \in \chi_{\text {back }}, K_{1}(X, t)=\alpha \cdot \cos \left(\frac{2 \pi}{T} t\right)
$$

where $T$ is the undulation period, allows to obtain upward and downward movements of the robot. Note that if the sine period isn't equal to the undulation period, this is will also induces a roll movement $\left(K_{1}\right.$ is defined in section 2).

- A sine pitch movement applied to the robot's back part :

$$
\forall X \in \chi_{\text {back }}, K_{2}(X, t)=\beta \cdot \cos \left(\frac{2 \pi}{T} t\right)
$$

produces a roll movement of the robot $\left(K_{2}\right.$ is defined in section 2). Indeed, if the the sine and the undulation periods are not equal, the behavior is not the same.

\section{STATEMENT OF THE CONTROL PROBLEM}

The control problem is schematically depicted on figure 5. A target position $P_{d}$ is given that is not necessarily in the transverse plane $\left(0, t_{10}, t_{20}\right)$ of the eel's mean line. The controller has to appropriately modify the control input :

$$
\mathbf{u}:=K(\cdot, \cdot)
$$

in order to steer the head $O$ towards the desired position $P_{d}$. Note however that we are not interested in point-wise stabilization at $P_{d}$ since the effectively used $P_{d}$ will be later generated by tele-operation mode and will be continuously moving. This is because the eel robot falls in the class of systems (including the rolling cart, the snakeboard, etc.) that cannot be controlled at zero-velocity. In addition to the control objective consisting in steering the eel's head to the desired position $P_{d}$, the rolling angle of the body has to be controlled. When the eel is in arbitrary $3 D$ configuration, expressing the desired rolling angle is not an easy task. Many choices can be done. Here, the controller is oriented towards the regulation of the scalar product :

$$
p_{\text {rol }}:=t_{20} \cdot E_{3}
$$




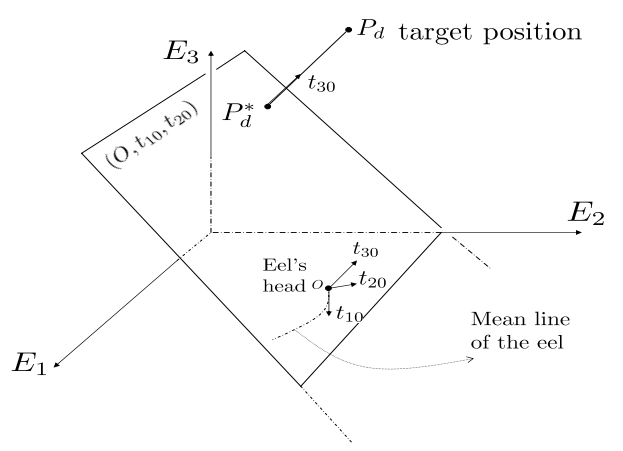

Fig. 5. Schematic 3D view of the control problem. The Eel that lies at instant $t$ in the plane $\left(0, t_{10}(t), t_{20}(t)\right)$ has to join the target position $P_{d}$ while trying to keep the vector $t_{20}$ horizontal, namely $t_{20} \cdot E_{3} \approx 0$. This has to be done using the infinite dimensional control input $K(\cdot, \cdot)$.

around 0 . Indeed, this guarantees that when the eel mean line lies in a horizontal plane, its transverse plane is also horizontal. Moreover, we look for a feedback law enabling the mean velocity of the head, namely $V_{0}=$ $\frac{1}{T} \int_{t-T}^{t}\left\|\dot{r}_{0}(\tau)\right\| d \tau$ to be controlled by appropriately controlling the characteristic of the body deformation through the control input $K(\cdot, \cdot)$.

\section{THE PROPOSED FEEDBACK}

As explained later, our aim is to realize the 3D control of the eel-like robot without using its pectoral fins. The idea is to control the head altitude by applying a sine twist movement to the robot's back part and to apply a sine pitch movement for rolling angle control.

The undulation laws $K_{1}$ and $K_{2}$ takes then the following form :

$$
\begin{aligned}
\forall X \in \chi_{\text {back }}, \quad K_{1}(X, t) & =\alpha \cdot \cos \left(\frac{2 \pi}{T} t\right) \\
K_{2}(X, t) & =\beta \cdot \cos \left(\frac{2 \pi}{T} t\right)
\end{aligned}
$$

where $\alpha \in\left[-\alpha_{\max }, \alpha_{\max }\right]$ and $\beta \in\left[-\beta_{\max }, \beta_{\max }\right]$ are used as control variables. $T$ is the undulation period.

The undulation law $K_{3}$ takes the following form in accordance with biological observations (Carling et al. [1998]) :

$$
K_{3}(t, X):=u_{3} \cdot A\left(X, u_{2}\right) \sin \left(\frac{X}{\lambda}-\frac{t}{T}\right)+u_{1}
$$

where $u_{3} \cdot A\left(X, u_{2}\right)$ gives the amplitude of the undulation as a function of the material abscissa $X$. The control input $u_{3} \in\left[0, u_{3}^{\max }\right]$ scales the amplitude in order to control the velocity. On the other hand, the control input $u_{2} \in\{-1,1\}$ defines whether the amplitude of undulations is bigger at the eel's tail or the eel's head. This is used to enhance acceleration or deceleration according to the velocity related control requirements.

The remaining control input $u_{1} \in\left[-u_{1}^{\max }, u_{1}^{\max }\right]$ is used to control the eel's movement in the transverse plane as it is explained in the following section.

Note that the velocity control and the control in the transverse plane are treated in our previous work (Alamir et al. [2007]). The control in the transverse plan will be represented here. For more information about velocity control, the reader may refer to (Alamir et al. [2007]). Note that (10)-(11)-(12) define a finite dimensioned parametrization of the control input leading to the control vector $\left(\alpha, \beta, u_{1}, u_{2}, u_{3}\right)$.

\subsection{Controlling the movement in the transverse plane $\left(0, t_{10}, t_{20}\right)$}

The way this is done is based on the following observation : When the undulation law (12) is used with $u_{1} \equiv 0$, a strait movement in the plane $\left(0, t_{10}, t_{20}\right)$ is asymptotically obtained while constant non vanishing values of $u_{1}$ asymptotically lead to circular trajectories.

Therefore, the control law uses $u_{1}$ to correct the direction of the movement in the plane $\left(0, t_{10}, t_{20}\right)$ in order to "move strait towards" the target position $P_{d}^{*}$. Note that $P_{d}^{*}$ is the projection parallel to $t_{30}$ of $P_{d}$ on the plane $\left(0, t_{10}, t_{20}\right)$ (see figure 5). The feedback uses the following key quantities :

$$
p_{e x t}:=-\pi_{3}\left(\overrightarrow{O P_{d}^{*}} \wedge t_{10}\right) \quad ; \quad p_{s c}=-\overrightarrow{O P_{d}^{*}} \cdot t_{10}
$$

where $\pi_{3}(\cdot)$ designates the third component of the argument. With the above notations, the following feedback is defined on $u_{1}$ :

$$
\begin{aligned}
u_{1}(t) & =F_{1}\left(\overrightarrow{O P_{d}^{*}}(t), t_{10}(t)\right) \\
& := \begin{cases}-\beta_{c} \cdot \bar{p}_{\text {ext }}(t) & \text { if } \bar{p}_{s c}(t)>0 \\
u_{1}^{\text {max }} & \text { otherwise }\end{cases}
\end{aligned}
$$

where $\bar{p}_{\text {ext }}(t)$ and $\bar{p}_{s c}(t)$ are the mean values of $p_{\text {ext }}$ and $p_{s c}$ over the past period of undulation, namely :

$$
\bar{p}_{\text {ext }}(t)=\frac{1}{T} \int_{t-T}^{t} p_{\text {ext }}(\tau) d \tau \quad ; \quad \bar{p}_{s c}(t)=\frac{1}{T} \int_{t-T}^{t} p_{s c}(\tau) d \tau(14)
$$

This amounts to stabilize $\bar{p}_{\text {ext }}$ to 0 with $\bar{p}_{s c}>0$ which means by definition of $p_{e x t}$ and $p_{s c}$ that $-t_{10}$ and $\overrightarrow{O P_{d}^{*}}$ are parallel (in average) and in the same direction. Once this is reached, $u_{1}$ vanishes and the eel adopts no mean curvature and moves towards the projected target $P_{d}^{*}$ with a strait mean line. The conditional use of $u_{1}=u_{1}^{\max }$ enables a maximal curvature to be used when the eel moves in the opposite direction to the one that would be necessary to move towards $P_{d}^{*}$ and since this corresponds to a circular trajectory, the sign of $p_{s c}$ necessarily becomes $>0$ after a finite time after which the above argumentation holds.

Note that during three dimensional manoeuvres, the transverse plane $\left(0, t_{10}, t_{20}\right)$ moves. This makes the projected position $P_{d}^{*}$ moving even for fixed target state $P_{d}$. Note that (13) is a proportional law, PID version can also be adopted.

\subsection{Controlling the altitude}

The solution proposed by Alamir et al. [2007] uses the robot's pectoral fins to control the following variable :

$$
z:=r_{0}(t) \cdot E_{3}
$$

around the desired value $z_{d}:=\pi_{3}\left(P_{d}\right)$. A robust sliding mode controller is used. 
Another approach that is more simple, completely independent of the robot's model and that can be used to control the altitude with or without using the robot's pectoral fins is presented here. This is done by controlling the following variable (see figure 5):

$$
d_{z}:=\overrightarrow{O P_{d}} \cdot t_{30}=P_{d}^{*} P_{d}
$$

around 0 . This amounts to move the transversal plane to contain $P_{d}$.

The design approximation is that the evolution of $d_{z}$ follows basically the following law :

$$
\ddot{d}_{z} \sim k_{z} \cdot \alpha
$$

where $\alpha$ (defined in (11)) is supposed to belong to $\left[-\alpha_{\max },+\alpha_{\max }\right]$. This suggests the following feedback law :

$$
\hat{\alpha}:=\alpha_{\max } \tanh \left(-\kappa_{1 a} d_{z}-\kappa_{2 a} \dot{d}_{z}\right)
$$

that is

$$
\hat{\alpha}:=\alpha_{\max } \tanh \left(-\kappa_{1 a} d_{z}-\kappa_{2 a}\left(\left(\omega_{0} \times t_{30}\right) \cdot \overrightarrow{O P_{d}}-V_{0} \cdot t_{30}\right)\right)
$$

where the use of the tanh function enables the saturation on $\alpha$ to be satisfied. Therefore, the control low uses $\alpha$ to correct the direction of the plane $\left(0, t_{10}, t_{20}\right)$ in order to cancel the distance $P_{d}^{*} P_{d}$.

\subsection{Controlling the rolling angle}

As indicated earlier, the control of the rolling angle amounts to control $p_{\text {rol }}:=t_{20} \cdot E_{3}$ around 0 . The same principle used in the altitude control is applied here.

The evolution of $p_{\text {rol }}$ follows basically the following law :

$$
\ddot{p}_{\text {rol }} \sim k_{\text {rol }} \cdot \beta \text {. }
$$

where $\beta$ (defined in (11)) is supposed to belong to $\left[-\beta_{\max },+\beta_{\max }\right]$. In the same way, this suggests the following feedback law :

$$
\begin{aligned}
\hat{\beta} & :=\beta_{\text {max }} \tanh \left(-\kappa_{1 r} p_{\text {rol }}-\kappa_{2 r} \dot{p}_{\text {rol }}\right) \\
& :=\beta_{\text {max }} \tanh \left(-\kappa_{1 r} p_{\text {rol }}-\kappa_{2 r} \pi_{3}\left(\omega_{0} \times t_{20}\right)\right)
\end{aligned}
$$

\section{NUMERICAL SIMULATIONS USING THE 3D CONTINUOUS MODEL OF BOYER ET AL. [2006]}

In this section, some numerical simulations (without velocity control) are proposed to assess the efficiency and underline some interesting features of the 3D movement control design.

\section{$6.13 D$ manoeuvres without velocity control}

The following simulations are proposed with constant values for the control inputs $u_{2}$ and $u_{3}$. Keeping these control inputs constant means that no feedback is done to control the mean velocity of the robot. This means that the robot cannot stop at the desired position. Once a neighborhood of the desired position is reached, the controlled robot can only "turn around" it waiting for the next set-point change. In what follows, some parameters used in the simulation are given.

\subsection{The robot parameters}

The exhaustive definition of the model parameters is given in (Boyer et al. [2006]). Let us mention here that the length of the robot is $L=2.08 \mathrm{~m}$ and all the cross sections are ellipsoidal with evolutive dimension that reproduces a quite realistic and faithful form (the tail is thinner that the central body).

\subsection{Control related parameters}

- The undulation period $T=1.2 s$ [see (12)]

- The wavelength $\lambda=1.3 \mathrm{~m}$

- The feedback gain $\beta_{c}=1$ [see (13)]

- The saturation level $u_{1}^{\max }=0.5$ [see (13)]

- Sampling period $\tau_{s}=0.1 \mathrm{~s}$

- The saturation on the twist angle $\alpha_{\max }=15 \mathrm{deg}$ [see (11)]

- The saturation on the pitch angle $\beta_{\max }=20 \mathrm{deg}$ [see (11)]

- The $z$ control parameters $\left(\kappa_{1 a}, \kappa_{2 a}\right)=(4,3)$ [see $\left.(17)\right]$

- The rolling angle feedback gains $\left(\kappa_{1 r}, \kappa_{2 r}\right)=(5,3)$ [see (19)]

- $\chi_{\text {back }}=[1.352, L]$ (35\% of the robot's length are used as the robot's back part)[see (8)].

\subsection{Manoeuvre description}

Three set-point changes are successively applied, each represents simultaneous changes in the three coordinates of the desired position $P_{d}$. The robot is initially at rest with the head at the origin "oriented" towards the negative values of $x$. The desired state is then defined by the following expression :

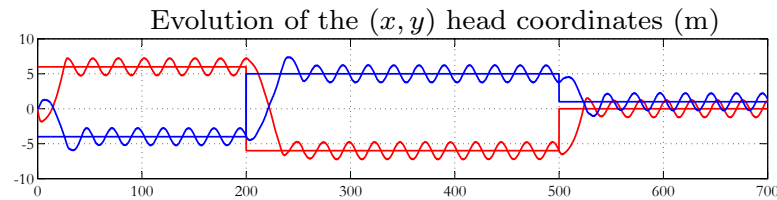

Evolution of the $(z)$ head coordinates $(\mathrm{m})$

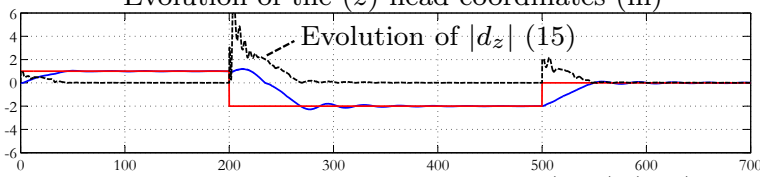

Evolution of the rolling angle $\arcsin \left(p_{\text {rol }}\right)(\mathrm{deg})$

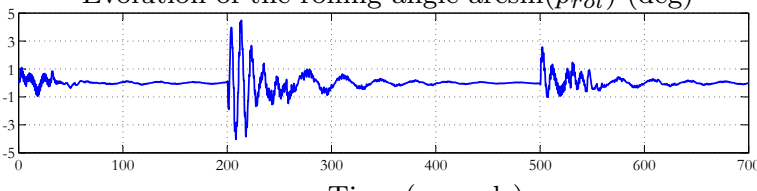

Time (seconds)

Fig. 6. Behavior of the controlled robot with the rolling angle control under the three successive set-point changes given by $(20)$

$$
P_{d}(t)= \begin{cases}(6,-4,1)^{T} & \text { for } t \leq 200 s \\ (-6,5,-2)^{T} & \text { for } 100<t \leq 500 s \\ (0,1,0)^{T} & \text { for } t>700 s\end{cases}
$$

Figure 6 shows the behavior of the head's coordinates as well as the evolution of the rolling angle $\arcsin \left(p_{\text {rol }}\right)$. The evolution of the control variable $\left(\alpha, \beta, u_{1}\right)$ during this 

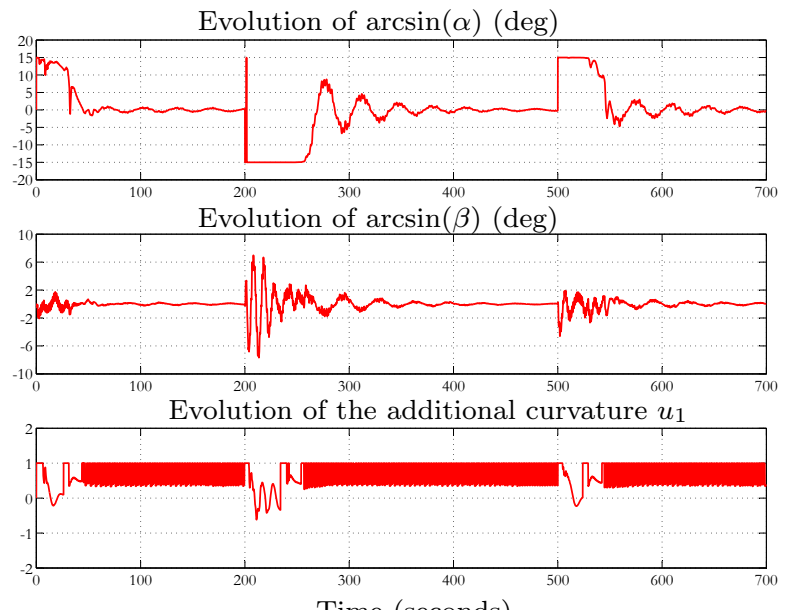

Time (seconds)

Fig. 7. Evolutions of the roll and pitch angles $(\alpha, \beta)$ as well as the additional curvature $u_{1}$ during the scenario of figure 6 .

scenario is depicted on figure 7 . Note that except for the transient phases that follows set-point changes, the control input $u_{1}$ makes the robot "turn around" the desired position $P_{d}$ since no velocity control is applied here.

Two videos could be seen at the bottom $\operatorname{link}^{2}$, they show the behavior of the simulator in tele-operation mode. Some keyboard keys are used by an operator in order to make the eel robot's model going through some virtual obstacles. A real time evolution is presented.

\section{CONCLUSION}

In this paper, preliminary results on the $3 \mathrm{D}$ control of an eel-like robot without pectoral fins are presented. We build on a previous work in which we proposed a complete control scheme for robot's 3D movement using its pectoral fins (Alamir et al. [2007]).The proposed feedback is quite simple and independent of the detailed structure of the robot's model. The controller is validated using the complete continuous model of Boyer et al. [2006]. Future work concerns the implementation on the prototype (under construction). For this, a systematic identification and tuning strategy need to be developed.

\section{REFERENCES}

M. Alamir, M. El Rafei, G. Hafidi, N. marchand, M. Porez, and F. Boyer. Feedback design for 3d movement of an eel-like robot. In Proceedings of the IEEE Int. Conf. Robotics and Automation, pages 256-261, Roma, 2007.

P. R. Bandyopadhyay. Trends in biorobotic autonomous undersea vehicles. IEEE Journal of Oceanic Engineering, 30(1):109-139, January 2005.

F. Boyer, M. Porez, and W. Khalil. Macro-continuous computed torque algorithm for a three-dimensional eel-like robot. IEEE Transaction on Robotics and Automation, 22(4):763-775, August 2006.

J. J. Burgess. Bending stiffness in a simulation of undersea cable deployment. Int. J. of Offshore and Polar Engineering, 3(3), 1993.

\footnotetext{
2 http://www.lag.ensieg.inpg.fr/alamir, link Projects
}

A. Cardona and M. Géradine. A beam finite element nonlinear theory with finite rotations. Int. J. Numer. Meth. Engng, 26:2403-2438, 1988.

J. Carling, T. L. Williams, and G. Bowtell. Self-propelled anguilliform swimming: simultaneous solution of the two-dimensional navier-stokes equations and newtons laws of motion. Journal of experimental biology, 201: 3143-3166, 1998.

J. E. Colgate and K. M. Lynch. Mechanics and control of swimming: A review. IEEE Journal of Oceanic Engineering, 29(3):660-73, July 2004.

R. Mason and J. W. Burdick. Experiments in carangiform robotic fish locomotion. In Proceedings of the IEEE Int. Conf. Robotics and Automation, pages 428-435, San Francisco, 2000.

K. A. McIsaac and J. P. Ostrowski. A geometric approach to anguiliform locomotion modelling of an underwater eel robot. In Proceedings of the IEEE Int. Conf. Robotics and Automation, pages 2843-2848, Detroit, 1999.

K. A. Morgansen, V. Duidam, R. J. Mason, J. W. Burdick, and R.M. Murray. Nonlinear control methods for planar carangiform robot fish locomotion. volume 1, pages 427434, 2001.

K. A. Morgansen, P. A. Vela, and J. W. Burdick. Trajectory stabilization for a planar carangiform robot fish. In Proceedings of the IEEE Int. Conf. Robotics and Automation, pages 756-762, Washington, 2002.

J. R. Morison. The force exerted by surface waves on piles. Transactions of the AIME, 189:149-154, 1950.

M. Sfakiotakis, D. M. Lane, and B. C. Davies. Review of fish swimming modes for aquatic locomotion. IEEE Journal of Oceanic Engineering, 24(2):237-252, April 1999.

J. C. Simo. A finite strain beam formulation. the three dimensional dynamic problem. part i: formulation and optimal parametrization. Comp. Meth. Appl. Mech. Eng., 72:276-304, 1989.

A. H. Techet and M. S. Triantafyllou. Fluid forces on bodies. Spring term, Ocean Engineering, MIT, 2004.

M. S. Triantafyllou and G. S. Triantafyllou. An efficient swimming machine. Scientific American, 272:64-70, March 1995.

M. S. Triantafyllou, G. S. Triantafyllou, and R. Gropalkrishnan. Optimal thrust development in oscillating foils with application to fish propulsion. J. Fluids Structures, 7:205-224, 1993.

P. A. Vela, K. A. Morgansen, and J. W. Burdick. Underwater locomotion from oscillatory shape deformations. In Proceedings of the 41st IEEE Conference on Decision and Control, pages 2074-2080, Las Vegas, 2002.

P.W. Webb. Form and function in fish swimming. Sci. Amer., 251:58-68, 1984.

J. Yu, L. Wang, and M. Tan. A framework for biomimetic robot fish's design and its realisation. In American Control Conference, pages 1593-1598, Portland, 2005. 